\title{
Parenting Skill Performances of Mothers of Young Children with Developmental Delay in Taiwan
}

\author{
Ming-Jae Lo \\ Department of Early Childhood Education \\ National Taichung University of Education \\ Taiwan (R.O.C.)
}

\begin{abstract}
The purpose of this study was to explore the different parenting skills of mothers in various demographics in parenting their young children with development delay. The participants were mothers of 3-6 year old children with development delay (DDC mothers) from a registered preschool in Taiwan. Mothers Parenting Skills Questionnaire (MPSQ) was used as the research tool. A convenience sampling of 199 valid questionnaires was collected. Nearly 80\% of the children exhibited developmental delay in language and communications; less prominent developmental delays were in (ranked in descending order by proportion) motor, cognitive, emotional, interpersonal, activities of daily living, and physiological aspects. The results of the present study revealed that the parenting skills of the DDC mothers approached the "moderately to satisfactorily met the criteria" level. The multiple regression analysis results revealed that the DDC mothers whose parenting skills were acquired from "parenting seminars" or "professionals or physicians," residing in northern Taiwan, and attaining an education level of university or above exhibited higher performance in overall parenting skills, achieving a statistically significant correlation. The research results can serve as a reference for the DDC mothers of young children, educator of preschools, and early-intervention facilities.
\end{abstract}

Keywords: mother of young children with development delay, education, information sources of parenting skills, urban-rural differences

\section{Introduction}

According to the Special Education Transmit Net (2018) database in Taiwan concerning families with young children with special needs, $80.89 \%$ of preschoolers with special needs exhibit developmental delay, with lower proportions exhibiting emotional behavioral disorders $(0.30 \%)$, visual impairments $(0.20 \%)$, and general learning disability $(0 \%)$. A national survey conducted in 2010 on the needs of 1,411 families with 5-year-old preschool children with physical or mental disabilities revealed that the forms of assistance most urgently required by parents were $42.6 \%$ "Understanding the means to educate and nurture their children" and 36.3\% "Understanding their children's current or future situation" (Special Needs Education Longitudinal Study, 2010).

Bailey and Simeonsson (1988) investigated 34 pairs of parents of infants with physical or mental disabilities and discovered that in the dimension of information needs, "education and nurture" was one of the aspects that parents needed the most. A survey on the needs of families with 0-6-year-old infants and young children with special needs revealed high demand in the following two items in the category of "Information needs": "Providing parental skills for educating and nurturing children at home" and "Providing information related to family or parent-children activities or workshops. (Cheng, 2011)" Family is the first and most effective nurturing context for young children with developmental delay, and parents are the primary teachers of such children. In addition, parental intervention is the longest lasting form of nurture and thus generates the strongest effect (Cheng, 2011; Lindsay \& Strand, 2013). Therefore, only when parents are equipped with adequate parenting skills can the needs of young children with developmental delay be satisfied and their development be accelerated.

According to the Certain Provisions in the Amendments For the Bye- laws of the Protection of Children; Youth Welfare and Rights Act (2015), "children with developmental delay" are defined as "children with suspected impairments or expected to develop impairments related to aspects such as cognitive development, physiological development, language and communication skills, psychological and social development, and self-care skills, and who have been evaluated in a hospital approved by the competent authority in charge of health care, confirmed as exhibiting such impairments, and issued a certificate." 
The following subsections detail nine principles proposed in parenting theories (Baumrind, 1967; Chen, Joseph, \& Wei, 2007; Erikson, 1963) and the maternal parenting skills evaluation tool developed by Lo, Lin and Kuo (2018) and discuss methods to improve maternal parenting skills for young children with developmental delay.

\section{Basic needs principle}

Compared with parents of healthy children, parents of young children with developmental delay require additional abilities to satisfy their children's various needs, including needs related to physiology, safety, socialization, respect, and self-actualization (Fenning, Baker, \& Juvonen, 2011; Goodlin-Jones, Tang, Liu, \& Anders, 2009). This gap is caused by young children with developmental delay falling behind their healthy counterparts in terms of cognitive development, gross and fine motor skills, and language delivery (Bruder, 1993; Fenning, et al., 2011; Tsai, 2009). In addition, children with developmental delay require increased rigidity in managing their dietary needs and sleep patterns and are more prone to emotional swings than their healthy counterparts are. Consequently, their parents require increased abilities in meeting their basic needs related to aspects such as feeding, sleep, daily schedule arrangement, and safety maintenance.

\section{Parenting preparation principle}

Parents who possess young children with developmental delay must be aware of the needs to "provide young children with stimuli" and "provide young children with toys" to educate and nurture them (Cheney, Manning \& Upham, 1997

; Cormany, 1992 ; Ramey \& Ramey, 1992). Caro and Derevensky (1991) surveyed parental education courses suitable for parents who possess young children with developmental delay and discovered that the top three course types that such parents were interested in were, in descending order, how to invoke learning motivation in children, how to assist children in building confidence, and how to assist children in intervention training. Therefore, before parents educate children, they should inspire their children's learning motivation and desire and prepare suitable teaching materials to assist their children in developing various abilities. The parenting preparation principle is one of the methods proposed in this study to increase the parenting skills of parents who possess young children with developmental delay.

\section{Generalization principle}

In early intervention for young children with developmental delay, the implementation of parental education is the most critical aspect to effectively facilitate the development of such children. Compared with early intervention professionals, parents possess more time to take care of, accompany, educate, and nurture their young children; therefore, they influence their children substantially (Lindsay, \& Strand, 2013; Williamson, 1997). Young children with developmental delay exhibit total or partial delay in their mental, social, emotional, language, or physiological development and commonly display lower development in these fields than their healthy counterparts (Goodlin-Jones, et al., 2009). Therefore, when selecting learning materials for children with developmental delay, parents must base their selection on the abilities that their children already possess and their children's current developmental level. Teaching materials should be arranged from basic to advanced.

\section{Initiative principle}

Influenced by the current low birth rate, many contemporary parents pay considerable attention to educating and nurturing their children; such parents have acquired the nickname "helicopter parents," (Fingerman, Cheng, Wesselmann, Zarit, Furstenberg, \& Birditt, 2012).And they tend to be overly anxious and involved in their children's lives, watching their children's every move at every instance as if from a helicopter. Consequently, children of such parents are deprived of opportunities to perform tasks independently; such deprivation can hinder the growth, adaptation, and development of young children with developmental delay. Therefore, when educating children with developmental delay, parents should encourage their children to perform tasks independently while providing patient coaching to cultivate independence, autonomy, and life skills in children.

\section{Personality adaptation and simultaneous learning principle}

Parents select teaching contents to provide the most satisfactory guidance to children according to their disability types, current cognitive development situations, hobbies, and talents. According to a study on the influence of home service on primary caregivers' methods (including attitudinal and behavioral aspects) for educating and nurturing young children with developmental delay, which takes the form of severe or explicit multiple disabilities and severe general learning disability, the factors to main caregivers' education and nurture attitudes were changes in the cognition related to the development history of children with developmental delay; respect for individual differences in providing diverse guidance for learning and growth; and understanding the importance of interaction modes (Yin, 2010). 
Because young children with developmental delay exhibit inferior cognitive abilities, motor skills, and social skills compared with their healthy counterparts, their parents may integrate knowledge, skills, and attitudes into learning activities according to their children's interests (Tsai, 2002).

\section{Socialization principle}

Children with developmental delay may encounter obstacles in social development because of their inferior social cognition, social skills (including responsibility, cooperation, self-control, self-respect, and self-affirmation), and emotional regulation compared with healthy young children (Bruder, 1993 ; Dettemer, Thurston \& Dyck,1993 ; Fenning, et al., 2011). According to Maccoby and Martin (1983), parents who educate their children should teach their children to comply with socialization rules and control their behaviors. Therefore, parents of young children with developmental delay should consider their children's abilities when presenting them with opportunities for group activities; parents should also cultivate favorable group living habits in said children such as respect for themselves and others and teach them how to cooperate with and help others.

\section{Gestalt principle}

In a survey of the educational needs of parents who possess children with developmental delay, cultivating cognitive ability, motor skills, and social skills in such children ranked second in terms of importance (Tsai,2002). Therefore, when parents teach such children, they should utilize authentic activities in various fields such as physical movement and health, cognition, communication and expression, society, emotions, and aesthetics to enable children to acquire a comprehensive set of life experience and undergo holistic development (Taiwan's Ministry of Education, 2016).

\section{Mastery principle}

Primary caregivers' education and nurture behaviors of demanding, responding, and using prompts influence the development of young children with developmental delay considerably (Yin, 2010). During the learning process of such children, parents provide children with adequate prompts and assistance and sufficient time for practice. If said children cannot meet the expected standards, they should be provided with additional prompts and chances to practice.

\section{Behavior guidance principle}

When parents of children with attention deficit hyperactivity disorder or emotional developmental delay do not understand the cause of their children's hyperactivity or emotional instability, they may consider their children as rebellious and intentionally challenging the authority (Finz-Dottan, Manor, \& Tyano, 2006). Consequently, said parents may adopt inappropriate strategies such as severe control, commands, and criticisms to alter their children's behavior. Therefore, the key to changing the problematic behaviors in children and increasing their educational and nurture satisfaction is to adopt appropriate education and nurture strategies and skills. Compared with healthy young children, those with developmental delay are substantially likelier to exhibit problematic behaviors (Baker et al., 2003; Borthwick-Duffy \& Eyman, 1990; Feldman, Hancock, Rielly, Minnes, \& Cairns, 2000; Steffenburg, Gillberg, \& Steffenburg, 1996; Stromme \& Diseth, 2000); such exhibition is often observed before school age (Baker et al., 2003; Feldman et al., 2000; Merrell \& Holland, 1997). Educational activities conducted by parents can assist children with developmental delay to achieve effective growth, adaptation, and development and promote societal integration among such children (Dettemer, et al., 1993). Therefore, parents of such children must equip themselves with appropriate behavioral coaching skills (e.g. using reinforcement or isolation techniques).

\section{Demographic characteristics and mothers' parenting skill performances}

Parenting skills or practices are likely to performance differently depending on cultural context and family or parental characteristics(Lo et al., 2018; Steinberg, Lamborn, Dornbusch, \& Darling, 1992).Taiwan survey results showed thata notable difference in parenting efficacy, attitudes, style, or skills among mothers in urban and rural areas (Chang, 2014; Li, 2003; Lo et al., 2018).

Asia studies have indicated causes of significantly different for young children mothers' parenting attitudes and perspectives or behaviors, such as their own age, and their children's age and sex (Chiu, 2017; Williams, Williams, Lopez, \& Tayko, 2000).Compared to young parents, older parents in United Statesof America tend to provide their children with more care and prompt reply to their needs (Weinberg \& Richardson, 1981). However, survey study found that the parenting skill performances demonstrated by Taiwanese mothers those of young children with typical development differed nonsignificantly in terms their own age, and their children's age and sex (Lo et al., 2018). As Taiwanese women's educational attainment continues to rise (Ministry of the Interior, R.O.C., 2018), its implications for parenting skills have been of great interest to researchers (Lo et al., 2018). 
The parental education needs for low-income parents of DDC in the Central Taiwan area can be divided into five aspects: "Young children development and education", "Professionals assistance and interaction", "Welfares and resources", "Self-adaptation and growth", "Family relationship and environmental improvement" (Lee, 2010). According to the concepts of social ecological models (Bronfenbrenner, 1979) and the empirical findings (Lee, 2010; Lo et al., 2018; Ma et al., 2012; Rowe, Barnes, \& Sutherns, 2013; Salonen, Kaunonen, Astedt-Kurki, Javenpaa, Isoaho, \& Tarkka, 2009), the present study divided the information sources of DDC maternal parenting skills into television (TV) media, broadcasts, newspapers and magazines, Internet, parenting seminars, relatives and friends, and professionals or physicians.

\section{Methods}

Parenting skills was assessed with the Mother Parenting Skills Questionnaire (MPSQ). MPSQ was a 4-point scale and divided into nine parenting skill dimensions. Answers "did not meet the criteria,"”moderately met the criteria," "satisfactorily met the criteria," and "excellently met the criteria" were given a score of 1, 2, 3, and 4, respectively. MPSQ yielded a Cronbach's $\alpha$ of .983, a test-retest showed a value of .892, and explained 70.02\% of the total variance (Lo et al., 2018). To assess the internal consistency of the MPSQ Scales in the entire sample, Cronbach's $\alpha$ were computed. Cronbach's $\alpha$ for the MPSQ meeting basic need, preparation, generalization, initiative, personality adaptation and simultaneous learning, mastery, socialization, Gestalt learning, behavioral counseling, and overall scale were $.860, .910, .888, .858, .940, .860, .855, .799, .788$, and .970 , respectively (Table 1 ).

Table 1 Reliability analysis of the mother parenting skills questionnaire (MPSQ) in DDC mothers ( $N=199)$

\begin{tabular}{lcc}
\hline MPSQ Subscale & Item N & Cronbach's $\alpha$ \\
\hline Meeting basic needs & 8 & .860 \\
Parenting preparation & 12 & .910 \\
Generalization & 8 & .888 \\
Initiative & 7 & .858 \\
Personality adaptations and simultaneous learning & 13 & .940 \\
Mastery & 4 & .860 \\
Socialization & 6 & .855 \\
Gestalt & 7 &. .799 \\
Behavioral counseling & 3 & .788 \\
Overall scale & 68 & .970 \\
\hline
\end{tabular}

\section{Official sample and sampling method}

This study recruited a sample of mothers of young children aged 3-6 with developmental delay attending one of public or private preschools in Northern, Central, Southern, and Eastern Taiwan. Convenience sampling was employed to recruit mothers of 3-6 years children with developmental delay for the official sample, with 60,60, 55, and 50(N=225) DCC mothers from Northern, Central, Southern, and Eastern Taiwan, respectively. A total of 207 questionnaires were returned (a return rate of 92\%), and 199 valid questionnaires (a valid return rate of 88.4\%) were obtained after excluding the invalid questionnaires because of incomplete or inconsistent responses.

\section{Statistical Analysis}

All data were coded, entered, and analyzed using Statistical Package for Social Sciences (SPSS Chinese Version 12.0). The descriptive results were expressed as numbers $(N)$, percentages $(\%)$, and means $(M) \pm$ standard deviation $(S D)$. Continuous variables were analyzed using an independent $t$ test or a one-way analysis of variance (ANOVA). The Scheffe test (homogeneity of variance) or the Games-Howell test (heterogeneity of variance)was performed as a posthoc test. Stepwise regression included regression models in which the predictive variables were selected using an automatic procedure. All results were considered statistically significant at $p<0.05$.

\section{Results}

\section{Demographic Characteristics of DDC mothers}

Table 2 indicated that DDC mothers, 28.6\%, 39.2\%, 17.6\%, and 14.6\% resided in Northern, Central, Southern, and Eastern Taiwan, respectively. Most of the children came from mother age of 31-35 years (35.2\%), 36-40 years (28.6\%) and 41-45 years (18.6\%); these three categories combined accounted for nearly $83 \%$ of all participants.

Most of the young children came from mother education of high school (31.7\%), university (27.1\%), college (26.1\%); these three categories combined accounted for nearly $85 \%$ of all participants. 
Most of the young children age came from 6 years (45.7\%), followed by those with 5 years (31.2\%); these two categories combined accounted for nearly $80 \%$ of all participants. Boys significantly outnumbered girls in the study, with 138 boys $(69.3 \%)$ and 61 girls $(30.7 \%)$.

Among DDC mothers, in terms of disability documents or other relevant documents of their young children, children who had a Comprehensive Evaluation Report of Children's Development (78.9\%) accounted for the majority, followed by those who had a Diagnostic Certificate (28.1\%), Disability Proof (27.6\%), and Major Injury and Disease Card $(6.0 \%)$.

Nearly $80 \%$ of the young children exhibited developmental delay in language and communications (76.9\%); less prominent developmental delays were in (ranked in descending order by proportion) motor (49.7\%), cognitive (43.7\%), emotional (35.7\%), interpersonal (34.7\%), activities of daily living (21.6\%), and physiological (10.6\%) aspects.

The information sources of parenting skill of DDC mothers, $56.8 \%, 52.3 \%, 38.2 \%, 36.7 \%, 29.1 \%, 23.1 \%$, and $4.0 \%$ came fromrelatives and friends, professionals or physicians, Internet, newspapers or magazines, parenting seminars, TV media, and broadcasts, respectively.

Table 2.Demographic characteristics and parenting related information of DDC mothers $(N=199)$

\begin{tabular}{|c|c|c|c|c|}
\hline DEMOGRAPHIC CHARACTERISTICS & GROUP & $\bar{N}$ & $\%$ & $R A N K$ \\
\hline \multirow{4}{*}{ 1.Mother residence } & Northern Taiwan & 57 & $28.6 \%$ & 2 \\
\hline & Central Taiwan & 78 & $39.2 \%$ & 1 \\
\hline & Southern Taiwan & 35 & $17.6 \%$ & 3 \\
\hline & Eastern Taiwan & 29 & $14.6 \%$ & 4 \\
\hline \multirow{6}{*}{ 2.Mother age } & 21-25 years & 2 & $1.0 \%$ & 6 \\
\hline & 26-30 years & 27 & $13.6 \%$ & 4 \\
\hline & 31-35 years & 70 & $35.2 \%$ & 1 \\
\hline & $36-40$ years & 57 & $28.6 \%$ & 2 \\
\hline & $41-45$ years & 37 & $18.6 \%$ & 3 \\
\hline & $46-50$ years & 6 & $3.0 \%$ & 5 \\
\hline \multirow[t]{5}{*}{ 3.Mother education level } & $\leqq$ Junior high school & 11 & $5.5 \%$ & 5 \\
\hline & High school & 63 & $31.7 \%$ & 1 \\
\hline & College & 52 & $26.1 \%$ & 3 \\
\hline & University & 54 & $27.1 \%$ & 2 \\
\hline & Master & 19 & $9.5 \%$ & 4 \\
\hline \multirow[t]{4}{*}{ 4.Young children age } & 3years & 6 & $3.0 \%$ & 4 \\
\hline & 4years & 40 & $20.1 \%$ & 3 \\
\hline & 5years & 62 & $31.2 \%$ & 2 \\
\hline & 6years & 91 & $45.7 \%$ & 1 \\
\hline \multirow[t]{2}{*}{ 5. Young children sex } & Boy & 138 & $69.3 \%$ & 1 \\
\hline & Girl & 61 & $30.7 \%$ & 2 \\
\hline \multicolumn{5}{|c|}{ In terms of disability documents or other relevant documents of their young children } \\
\hline \multirow{2}{*}{$\begin{array}{l}\text { 1.Comprehensive evaluation } \\
\text { report of children's development }\end{array}$} & Yes & 157 & $78.9 \%$ & 1 \\
\hline & No & 42 & $21.1 \%$ & \\
\hline \multirow[t]{2}{*}{ 2.Diagnostic certificate } & Yes & 56 & $28.1 \%$ & 2 \\
\hline & No & 143 & $71.9 \%$ & \\
\hline \multirow[t]{2}{*}{ 3.Disability proof } & Yes & 55 & $27.6 \%$ & 3 \\
\hline & No & 144 & $72.4 \%$ & \\
\hline \multirow[t]{2}{*}{ 4.Major injury and disease card } & Yes & 12 & $6.0 \%$ & 4 \\
\hline & No & 187 & $94.0 \%$ & \\
\hline \multicolumn{5}{|c|}{ Young children exhibited developmental delay } \\
\hline \multirow[t]{2}{*}{ 1.Language and communications delays } & Yes & 153 & $76.9 \%$ & 1 \\
\hline & No & 46 & $23.1 \%$ & \\
\hline \multirow[t]{2}{*}{ 2.Motor delays } & Yes & 99 & $49.7 \%$ & 2 \\
\hline & No & 100 & $50.3 \%$ & \\
\hline
\end{tabular}




\begin{tabular}{llrrr} 
3.Cognitive delays & Yes & 87 & $43.7 \%$ & 3 \\
4.Emotional delays & No & 112 & $56.3 \%$ & \\
& Yes & 71 & $35.7 \%$ & 4 \\
5.Interpersonal delays & No & 128 & $64.3 \%$ & 5 \\
& Yes & 69 & $34.7 \%$ & 5 \\
6.Activities of daily living delays & No & 130 & $65.3 \%$ & 6 \\
7.Physiological delays & Yes & 43 & $21.6 \%$ & 7 \\
Parenting related information & No & 156 & $78.4 \%$ & \\
1.Relatives and friends & Yes & 21 & $10.6 \%$ & \\
& No & 178 & $89.4 \%$ & 1 \\
2.Professionals or physicians & & & & \\
3.Internet & Yes & 113 & $56.8 \%$ & \\
& No & 86 & $43.2 \%$ & \\
4.Newspapers or magazines & Yes & 104 & $52.3 \%$ & \\
& No & 95 & $47.7 \%$ & 3 \\
5.Parenting seminars & Yes & 76 & $38.2 \%$ & \\
6.TV media & No & 123 & $61.8 \%$ & \\
7.Broadcasts & Yes & 73 & $36.7 \%$ & 4 \\
& No & 126 & $63.3 \%$ & 5 \\
\hline
\end{tabular}

\section{Analyzing DDC mothers' performance in nine parenting skills}

Among all nine skills, these mothers' skills for meeting children's basic needs were excellent $(M=3.17)$. In descending order of excellence, these mothers also possessed skills related to the behavioral counseling $(M=2.93)$, generalization $(M=2.83)$, mastery $(M=2.81)$, personality adaptations and simultaneous learning $(M=2.77)$, socialization $(M=2.76)$, initiative $(M=2.74)$, and preparation for parenting $(M=2.71)$. Among the nine parenting skills, the skill related to the Gestalt learning $(M=2.60)$ was the most unsatisfactory; however, the score on this skill was higher than the median (2.5 points) was, between moderately and satisfactorily met the criteria (Table 3 ).

Table 3 Situation analysis of DDC mothers' performance in MPSQ $(N=199)$

\begin{tabular}{|c|c|c|c|c|c|c|c|c|}
\hline \multirow{2}{*}{ MPSQ Dimensions } & \multicolumn{3}{|c|}{ Subscale } & \multicolumn{2}{|c|}{ Item } & \multirow{2}{*}{$\begin{array}{c}\text { Excellently Met The } \\
\text { Criteria } \\
\text { Percentage }\end{array}$} & \multirow[t]{2}{*}{ Rank } & \multirow{2}{*}{$\begin{array}{c}\text { Parenting } \\
\text { Levels }\end{array}$} \\
\hline & $\bar{N}$ & $M$ & $\overline{S D}$ & $M$ & $\overline{S D}$ & & & \\
\hline Meeting basic needs & 8 & 25.36 & 3.36 & 3.17 & 0.42 & 79.25 & 1 & $\bar{M}$ \\
\hline Behavioral counseling & 3 & 8.80 & 1.67 & 2.93 & 0.55 & 67.75 & 2 & L-M \\
\hline Generalization & 8 & 22.70 & 4.03 & 2.83 & 0.50 & 70.75 & 3 & L-M \\
\hline Mastery & 4 & 11.26 & 2.04 & 2.81 & 0.51 & 68.50 & 4 & L-M \\
\hline $\begin{array}{l}\text { Personality adaptations a } \\
\text { simultaneous learning }\end{array}$ & 13 & 36.02 & 6.56 & 2.77 & 0.50 & 65.00 & 5 & L-M \\
\hline Socialization & 6 & 16.61 & 3.32 & 2.76 & 0.55 & 69.25 & 6 & L-M \\
\hline Initiative & 7 & 19.23 & 3.45 & 2.74 & 0.49 & 70.25 & 7 & L-M \\
\hline Parenting preparation & 12 & 32.62 & 5.84 & 2.71 & 0.48 & 69.00 & 8 & L-M \\
\hline Gestalt & 7 & 18.25 & 1.84 & 2.60 & 0.26 & 73.25 & 9 & L-M \\
\hline Overall scale & 68 & 190.88 & 26.00 & 2.80 & 0.38 & 70.00 & & L-M \\
\hline
\end{tabular}


Item $M=$ subscale $M \div$ subscale $N$. The MPSQ was a 4-point scale. Answers "did not meet the criteria,"” moderately met the criteria," "satisfactorily met the criteria," and "excellently met the criteria" were given a score of 1,2, 3, and 4, respectively. Parenting skills were classified into three levels: requires improvement (Low), average (Middle), and excellent (High).

\section{Demographic characteristics and DDC mothers' performance in nine parenting skills}

There were no significant differences between mothers ages, young children age and sex for nine and overall parenting skills of DDC mother. The results showed that the generalization $(F=3.09, p<.05)$, socialization $(F=2.83, p<.05)$, personality adaptations and simultaneous learning $(F=4.00, p<.01)$, and overall parenting skills $(F=3.04, p<.05)$ of mothers in northern Taiwan were significantly superior than those of mothers in eastern Taiwan. The results showed that meeting the Gestalt $(F=2.87, p<.05)$, and overall parenting skills $(F=3.04, p<.05)$ of mothers in northern Taiwan were significantly superior than those of mothers in southern Taiwan. Mothers who had a university degree or a higher educational level exhibited significant better meeting children's basic needs $(F=4.92, p<.01)$, generalization $(F=6.89, p$ $<.01)$, initiative $(F=3.05, p<.05)$, personality adaptations and simultaneous learning $(F=5.38, p<.01)$, mastery $(F=3.12, p<.05)$, as well as overall performance $(F=5.08, p<.01)$ than mothers who had a high school (or a lower educational level), or college (Table 4).

Table 4Association between demographic variables and performance of DDC mothers parenting skills $(N=199)$

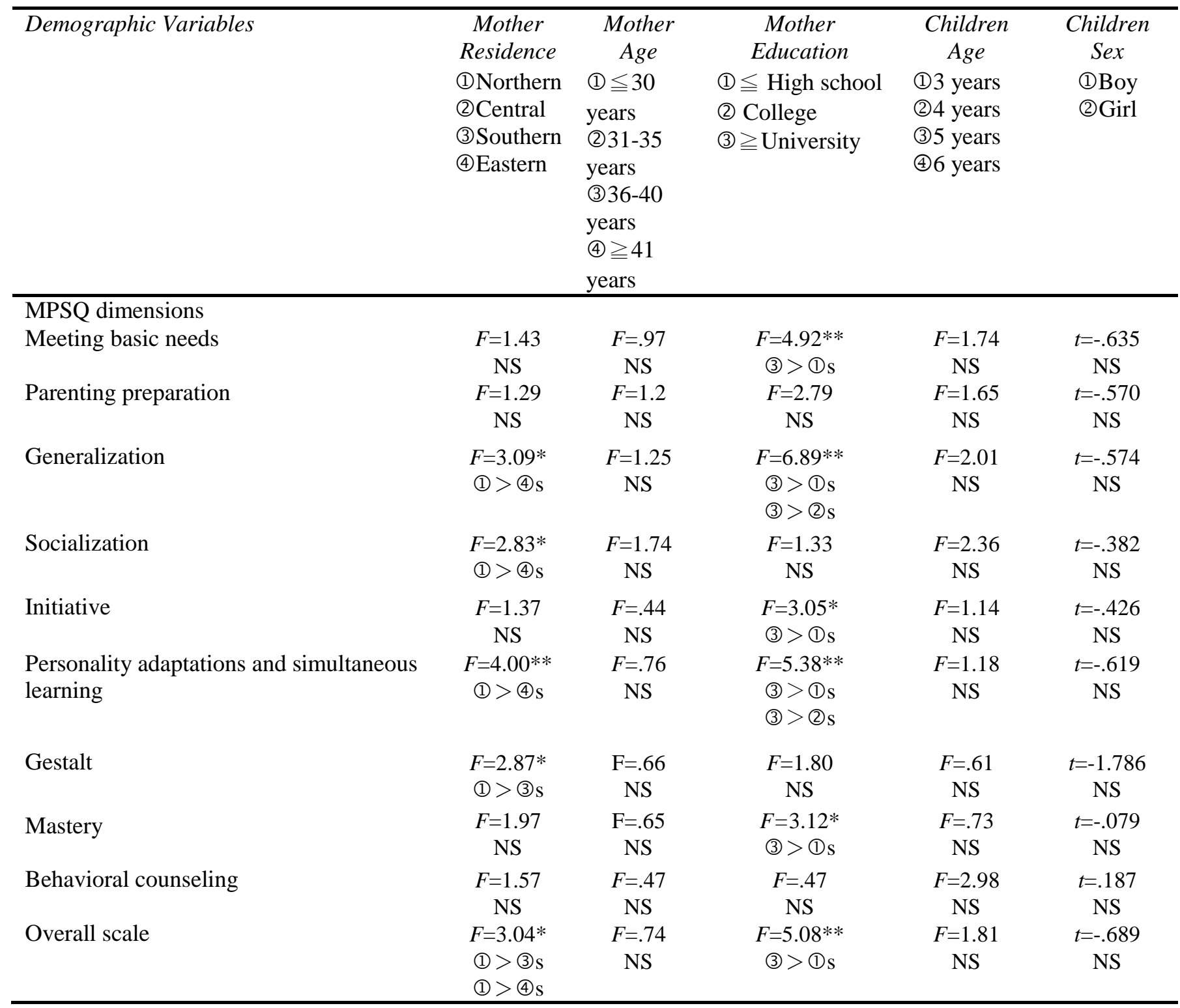

Notes: $* p<.05 . * * p<.01 . * * * p<.001 ;$ s-Scheffe; g- Games-Howell; NS, no significant. 
Association between types of young children with developmental delay and performance of DDC mothers parenting skills

Table 5 showed that the parenting skills of preparation $(t=-2.09, p<.05)$ and generalization $(t=-2.56, p<.05)$ incognitive DDC mothers were significantly inferior than those of non cognitive DDC mothers. However, the remaining seven skills, as well as overall performance were not significantly different in DDC mother who did not and did of young children exhibited cognitive delay. The parenting skills of socialization $(t=-2.38, p<.05)$ in emotional DDC mothers were significantly inferior than those of non emotional DDC mothers. The parenting skills of initiative $(t=-2.02, p<.05)$ in activities of daily living DDC mothers were significantly inferior than those of non activities of daily living DDC mothers.

Table 5 Association between types of young children with developmental delay and performance of DDC mothers parenting skills $(N=199)$

\begin{tabular}{|c|c|c|c|c|c|c|c|}
\hline Types of $D D$ & $\begin{array}{l}\text { Language and } \\
\text { communi- } \\
\text { cations } \\
\text { (1) Yes } \\
(N=46) \\
\text { (2) No } \\
(N=153)\end{array}$ & $\begin{array}{l}\text { (1) Yes } \\
(N=8) \\
\text { (2) No } \\
(N=191\end{array}$ & $\begin{array}{l}\text { (1) Yes } \\
(N=73) \\
\text { (2) No } \\
(N=126)\end{array}$ & $\begin{array}{l}\text { (1) Yes } \\
(N=76) \\
\text { (2) No } \\
(N=123)\end{array}$ & $\begin{array}{l}\text { (1) Yes } \\
(N=58) \\
\text { (2) No } \\
(N=141)\end{array}$ & $\begin{array}{l}\text { (1) Yes } \\
(N=113) \\
\text { (2) No } \\
(N=86)\end{array}$ & $\begin{array}{l}\text { (1) Yes } \\
(N=104) \\
\text { (2) No } \\
(N=95)\end{array}$ \\
\hline MPSQ dimensions & & & & & & & \\
\hline Meeting & $t=.58$ & $t=-.33$ & $t=-1.51$ & $t=-1.26$ & $t=-.18$ & $t=-.34$ & $t=-.178$ \\
\hline basic needs & NS & NS & NS & NS & NS & NS & NS \\
\hline Parenting preparation & $\begin{array}{c}t=-.87 \\
N S\end{array}$ & $\begin{array}{c}t=-.10 \\
\mathrm{NS}\end{array}$ & $\begin{array}{c}t=-2.09 * \\
\text { (2) }>\text { (1) }\end{array}$ & $\begin{array}{c}t=-.72 \\
\mathrm{NS}\end{array}$ & $\begin{array}{c}t=-.98 \\
\mathrm{NS}\end{array}$ & $\begin{array}{c}t=-.74 \\
\mathrm{NS}\end{array}$ & $\begin{array}{c}t=-1.04 \\
\mathrm{NS}\end{array}$ \\
\hline Generalization & $\begin{array}{c}t=-.59 \\
\mathrm{NS}\end{array}$ & $\begin{array}{c}t=-.69 \\
\text { NS }\end{array}$ & $\begin{array}{c}t=-2.56^{*} \\
\text { (2) }>\text { (1) }\end{array}$ & $\begin{array}{c}t=-1.92 \\
\mathrm{NS}\end{array}$ & $\begin{array}{c}t=-1.02 \\
\mathrm{NS}\end{array}$ & $\begin{array}{c}t=-1.03 \\
\mathrm{NS}\end{array}$ & $\begin{array}{c}t=-1.54 \\
\mathrm{NS}\end{array}$ \\
\hline Socialization & $\begin{array}{c}t=.98 \\
\mathrm{NS}\end{array}$ & $\begin{array}{c}t=-1.85 \\
\mathrm{NS}\end{array}$ & $\begin{array}{c}t=-.85 \\
\mathrm{NS}\end{array}$ & $\begin{array}{c}t=-2.38 * \\
\text { (2) }>\text { (1) }\end{array}$ & $\begin{array}{c}t=-1.24 \\
\mathrm{NS}\end{array}$ & $\begin{array}{c}t=--1.27 \\
\mathrm{NS}\end{array}$ & $\begin{array}{c}t=-1.11 \\
\mathrm{NS}\end{array}$ \\
\hline Initiative & $\begin{array}{c}t=-.25 \\
\mathrm{NS}\end{array}$ & $\begin{array}{c}t=-1.79 \\
\mathrm{NS}\end{array}$ & $\begin{array}{c}t=-1.52 \\
\mathrm{NS}\end{array}$ & $\begin{array}{c}t=-1.02 \\
\mathrm{NS}\end{array}$ & $\begin{array}{c}t=-1.22 \\
\mathrm{NS}\end{array}$ & $\begin{array}{c}t=-2.02 * \\
\text { (2) }>\text { (1) }\end{array}$ & $\begin{array}{c}t=-.80 \\
\mathrm{NS}\end{array}$ \\
\hline $\begin{array}{l}\text { Personality } \\
\text { adaptations and } \\
\text { simultaneous } \\
\text { learning }\end{array}$ & $\begin{array}{c}t=.88 \\
\text { NS }\end{array}$ & $\begin{array}{c}t=-.79 \\
\text { NS }\end{array}$ & $\begin{array}{c}t=-.77 \\
\mathrm{NS}\end{array}$ & $\begin{array}{c}t=-.94 \\
\text { NS }\end{array}$ & $\begin{array}{c}t=-1.04 \\
\mathrm{NS}\end{array}$ & $\begin{array}{c}t=-.58 \\
\mathrm{NS}\end{array}$ & $\begin{array}{c}t=.23 \\
\text { NS }\end{array}$ \\
\hline Gestalt & $\begin{array}{c}t=.32 \\
\mathrm{NS}\end{array}$ & $\begin{array}{c}t=-.45 \\
\mathrm{NS}\end{array}$ & $\begin{array}{c}t=-.61 \\
\mathrm{NS}\end{array}$ & $\begin{array}{c}t=-1.60 \\
\mathrm{NS}\end{array}$ & $\begin{array}{c}t=-1.65 \\
\mathrm{NS}\end{array}$ & $\begin{array}{c}t=-.45 \\
\mathrm{NS}\end{array}$ & $\begin{array}{c}t=1.52 \\
\mathrm{NS}\end{array}$ \\
\hline Mastery & $\begin{array}{c}t=1.15 \\
\mathrm{NS}\end{array}$ & $\begin{array}{c}t=-.41 \\
\mathrm{NS}\end{array}$ & $\begin{array}{c}t=-.19 \\
\mathrm{NS}\end{array}$ & $\begin{array}{c}t=-1.42 \\
\mathrm{NS}\end{array}$ & $\begin{array}{c}t=-.58 \\
\mathrm{NS}\end{array}$ & $\begin{array}{c}t=-1.03 \\
\mathrm{NS}\end{array}$ & $\begin{array}{c}t=-.28 \\
\mathrm{NS}\end{array}$ \\
\hline $\begin{array}{l}\text { Behavioral } \\
\text { counseling }\end{array}$ & $\begin{array}{c}t=-.70 \\
\mathrm{NS}\end{array}$ & $\begin{array}{c}t=-.22 \\
\mathrm{NS}\end{array}$ & $\begin{array}{c}t=-.34 \\
\mathrm{NS}\end{array}$ & $\begin{array}{c}t=-1.07 \\
\mathrm{NS}\end{array}$ & $\begin{array}{c}t=-.04 \\
\text { NS }\end{array}$ & $\begin{array}{c}t=-.88 \\
\mathrm{NS}\end{array}$ & $\begin{array}{c}t=-1.79 \\
\mathrm{NS}\end{array}$ \\
\hline Overall scale & $\begin{array}{c}t=.18 \\
\text { NS }\end{array}$ & $\begin{array}{c}t=-.92 \\
\text { NS }\end{array}$ & $\begin{array}{c}t=-1.64 \\
\mathrm{NS}\end{array}$ & $\begin{array}{c}t=-1.60 \\
\mathrm{NS}\end{array}$ & $\begin{array}{c}t=-1.15 \\
\mathrm{NS}\end{array}$ & $\begin{array}{c}t=-1.27 \\
\mathrm{NS}\end{array}$ & $\begin{array}{c}t=-.68 \\
\text { NS }\end{array}$ \\
\hline
\end{tabular}

Notes: ${ }^{*} p<.05 ;$ NS, no significant.

Sources of parenting skill information and performance of DDC mother parenting skills

DDC mothers parenting skills acquiring from TV media, broadcasts, newspapers or magazines, internet, relatives and friends were not correlated to nine and overall parenting skills performance. DDC mothers that did acquire parenting skills from parenting seminars, professionals or physicians performed in nine parenting skills and their overall performance were significant better than that did not acquire parenting skills from parenting seminars, professionals or physicians (Table 6). 
Table 6Association between parenting related information and performance of DDC mothers parenting skills $(N$ = 199)

\begin{tabular}{|c|c|c|c|c|c|c|c|}
\hline $\begin{array}{l}\text { Source of } \\
\text { parenting skill } \\
\text { Item }\end{array}$ & $\begin{array}{l}T V \\
\text { media } \\
\\
\text { (1) Yes } \\
(N=46) \\
(2) \mathrm{No} \\
(N=153)\end{array}$ & $\begin{array}{l}\text { (1) Yes } \\
(N=8) \\
\text { (2) No } \\
(N=191)\end{array}$ & $\begin{array}{l}\text { Newspapers } \\
\text { or magazines } \\
\text { (1)Yes } \\
(N=73) \\
(2 \mathrm{No} \\
(N=126)\end{array}$ & $\begin{array}{l}\text { (1) Yes } \\
(N=76) \\
\text { (2) No } \\
(N=123)\end{array}$ & $\begin{array}{l}\text { Parenting } \\
\text { seminars } \\
\text { (1) Yes } \\
(N=58) \\
\text { (2) No } \\
(N=141)\end{array}$ & $\begin{array}{l}\text { Relatives } \\
\text { or } \\
\text { friends } \\
\text { (1)Yes } \\
(N=113) \\
(2) \mathrm{No} \\
(N=86)\end{array}$ & $\begin{array}{l}\text { Professionals } \\
\text { or } \\
\text { physicians } \\
\text { (1) Yes } \\
(N=104) \\
(2 \mathrm{No} \\
(N=95)\end{array}$ \\
\hline $\begin{array}{l}\text { MPSQ dimensi } \\
\text { Meeting } \\
\text { basic needs }\end{array}$ & $\begin{array}{c}t=-.97 \\
\mathrm{NS}\end{array}$ & $\begin{array}{c}t=.44 \\
\mathrm{NS}\end{array}$ & $\begin{array}{c}t=1.73 \\
\mathrm{NS}\end{array}$ & $\begin{array}{c}t=1.45 \\
\mathrm{NS}\end{array}$ & $\begin{array}{c}t=2.93 * * \\
\text { (1) }>\text { (2) }\end{array}$ & $\begin{array}{c}t=-1.10 \\
\mathrm{NS}\end{array}$ & $\begin{array}{c}t=2.85^{* *} \\
\text { (1) }>\text { (2) }\end{array}$ \\
\hline $\begin{array}{l}\text { Parenting } \\
\text { preparation }\end{array}$ & $\begin{array}{c}t=-.11 \\
\mathrm{NS}\end{array}$ & $\begin{array}{c}t=.18 \\
\mathrm{NS}\end{array}$ & $\begin{array}{c}t=.1 .03 \\
\mathrm{NS}\end{array}$ & $\begin{array}{c}t=1.05 \\
\mathrm{NS}\end{array}$ & $\begin{array}{c}t=3.86^{* * * *} \\
\text { (1)> (2) }\end{array}$ & $\begin{array}{c}t=-1.68 \\
\mathrm{NS}\end{array}$ & $\begin{array}{c}t=3.67 * * * \\
\text { (1) > (2) }\end{array}$ \\
\hline Generalization & $\begin{array}{c}t=-.02 \\
\mathrm{NS}\end{array}$ & $\begin{array}{c}t=-.32 \\
\mathrm{NS}\end{array}$ & $\begin{array}{c}t=1.56 \\
\mathrm{NS}\end{array}$ & $\begin{array}{c}t=1.17 \\
\mathrm{NS}\end{array}$ & $\begin{array}{c}t=4.73 * * * \\
\text { (1) }>\text { (2) }\end{array}$ & $\begin{array}{c}t=-1.84 \\
\mathrm{NS}\end{array}$ & $\begin{array}{c}t=3.74 * * * \\
\text { (1) }>\text { (2) }\end{array}$ \\
\hline Socialization & $\begin{array}{c}t=1.70 \\
\mathrm{NS}\end{array}$ & $\begin{array}{c}t=-.97 \\
\mathrm{NS}\end{array}$ & $\begin{array}{c}t=1.45 \\
\mathrm{NS}\end{array}$ & $\begin{array}{c}t=.87 \\
\text { NS }\end{array}$ & $\begin{array}{c}t=2.72 * * \\
\text { (1) }>\text { (2) }\end{array}$ & $\begin{array}{c}t=-.04 \\
\mathrm{NS}\end{array}$ & $\begin{array}{c}t=3.06^{* *} \\
\text { (1) }>\text { (2) }\end{array}$ \\
\hline Initiative & $\begin{array}{c}t=.35 \\
\text { NS }\end{array}$ & $\begin{array}{c}t=-1.14 \\
\mathrm{NS}\end{array}$ & $\begin{array}{c}t=1.74 \\
\mathrm{NS}\end{array}$ & $\begin{array}{c}t=.72 \\
\text { NS }\end{array}$ & $\begin{array}{c}t=4.41 * * * \\
\text { (1) }>\text { (2) }\end{array}$ & $\begin{array}{c}t=-.73 \\
\mathrm{NS}\end{array}$ & $\begin{array}{c}t=5.48 * * * \\
\text { (1) }>\text { (2) }\end{array}$ \\
\hline $\begin{array}{l}\text { Personality } \\
\text { adaptations and } \\
\text { simultaneous } \\
\text { learning }\end{array}$ & $\begin{array}{c}t=-.13 \\
\mathrm{NS}\end{array}$ & $\begin{array}{c}t=-.34 \\
\mathrm{NS}\end{array}$ & $\begin{array}{c}t=1.85 \\
\mathrm{NS}\end{array}$ & $\begin{array}{c}t=.1 .17 \\
\mathrm{NS}\end{array}$ & $\begin{array}{c}t=4.20 * * * \\
\text { (1) }>\text { (2) }\end{array}$ & $\begin{array}{c}t=-1.07 \\
\mathrm{NS}\end{array}$ & $\begin{array}{c}t=4.10^{* * * *} \\
\text { (1) > (2) }\end{array}$ \\
\hline Gestalt & $\begin{array}{c}t=-.96 \\
\mathrm{NS}\end{array}$ & $\begin{array}{c}t=-.19 \\
\mathrm{NS}\end{array}$ & $\begin{array}{c}t=.37 \\
\text { NS }\end{array}$ & $\begin{array}{c}t=-1.83 \\
\mathrm{NS}\end{array}$ & $\begin{array}{c}t=2.79 * * \\
\text { (1) }>\text { (2) }\end{array}$ & $\begin{array}{c}t=-.63 \\
\text { NS }\end{array}$ & $\begin{array}{c}t=2.89 * * \\
\text { (1) }>\text { (2) }\end{array}$ \\
\hline Mastery & $\begin{array}{c}t=-.41 \\
\mathrm{NS}\end{array}$ & $\begin{array}{c}t=-1.07 \\
\mathrm{NS}\end{array}$ & $\begin{array}{c}t=1.58 \\
\mathrm{NS}\end{array}$ & $\begin{array}{c}t=1.15 \\
\mathrm{NS}\end{array}$ & $\begin{array}{c}t=2.62 * \\
\text { (1) }>\text { (2) }\end{array}$ & $\begin{array}{c}t=-1.16 \\
\mathrm{NS}\end{array}$ & $\begin{array}{c}t=3.18 * * \\
\text { (1) }>\text { (2) }\end{array}$ \\
\hline $\begin{array}{l}\text { Behavioral } \\
\text { counseling }\end{array}$ & $\begin{array}{c}t=-.50 \\
\mathrm{NS}\end{array}$ & $\begin{array}{c}t=.55 \\
\mathrm{NS}\end{array}$ & $\begin{array}{c}t=.55 \\
\mathrm{NS}\end{array}$ & $\begin{array}{c}t=.86 \\
\text { NS }\end{array}$ & $\begin{array}{c}t=2.59 * \\
\text { (1) }>\text { (2) }\end{array}$ & $\begin{array}{c}t=-1.62 \\
\mathrm{NS}\end{array}$ & $\begin{array}{c}t=2.70 * * \\
\text { (1) }>\text { (2) }\end{array}$ \\
\hline Overall scale & $\begin{array}{c}t=.19 \\
\text { NS }\end{array}$ & $\begin{array}{c}t=-.38 \\
\mathrm{NS}\end{array}$ & $\begin{array}{c}t=1.76 \\
\mathrm{NS}\end{array}$ & $\begin{array}{c}t=1.34 \\
\mathrm{NS}\end{array}$ & $\begin{array}{c}t=4.61 * * * \\
\text { (1) }>\text { (2) }\end{array}$ & $\begin{array}{c}t=-1.53 \\
\mathrm{NS}\end{array}$ & $\begin{array}{c}t=4.62 * * * * \\
\text { (1) }>\text { (2) }\end{array}$ \\
\hline
\end{tabular}

Notes: $* p<.05 . * * p<.01 . * * * p<.001$; A higher score indicated a better performance of parenting skill.

Abbreviations: NS, no significant.

Demographic characteristics, types of young children exhibited developmental delay, and information sources of parenting skill as predictors of DDC mothers parenting skills

A stepwise multiple regression analysis showed that DDC mothers who acquired parenting skills from professionals or physicians, and parenting seminar performed better nine and overall parenting skills. DDC mothers who had a University's degree or a higher educational level, performed better in meeting their children's basic needs $(\beta=.182)$, generalization $(\beta=.182)$, initiative $(\beta=.159)$, personalized adaptation and simultaneous learning skills $(\beta=.151)$, mastery $(\beta=.187)$, and overall performance $(\beta=.142)$.

Cognitive DDC mothers performed worse in parenting skills of preparation $(\beta=-.198)$, and generalization $(\beta=-.148)$. Emotional DDC mothers performed worse in parenting skill of socialization $(\beta=-.198)$. Activities of daily living DDC mothers performed worse in parenting skill of initiative $(\beta=-.133)$.

DDC mothers in eastern Taiwan performed worse than those of DDC mothers in northern Taiwan in parenting skills of generalization $(\beta=-.134)$, socialization $(\beta=-.197)$, personalized adaptation and simultaneous learning skills $(\beta=-.191)$, and overall performance $(\beta=-.186)$. DDC mothers in southern Taiwan performed worse than those ofDDC mothers in northern Taiwan in parenting skills of Gestalt $(\beta=-.154)$, and overall performance $(\beta=-.167)$ (Table 7). 
Table 7Demographic variables and performance of DDC mothers parenting skills: A stepwise multiple regression analysis $(N=199)$

\begin{tabular}{|c|c|c|c|c|c|c|c|}
\hline $\begin{array}{l}M P S Q \\
\text { Dimensions } \\
\end{array}$ & Variables & $\bar{R}$ & $\overline{R^{2}}$ & $\begin{array}{c}R^{2} \\
\text { change }\end{array}$ & $\bar{F}$ & $\beta$ & $\bar{t}$ \\
\hline \multirow{3}{*}{$\begin{array}{l}\text { Meeting basic } \\
\text { needs }\end{array}$} & $\geqq$ University degree $^{1}$ & .207 & .043 & .043 & $8.80^{* *}$ & .182 & $2.62 * *$ \\
\hline & Professionals or physicians ${ }^{2}$ & .268 & .072 & .029 & $6.18 *$ & .173 & $2.49 *$ \\
\hline & Parenting seminar ${ }^{3}$ & .287 & .092 & .020 & $5.74 *$ & .169 & $2.34 *$ \\
\hline \multirow{3}{*}{$\begin{array}{l}\text { Parenting } \\
\text { preparation }\end{array}$} & Parenting seminar $^{3}$ & .265 & .070 & .070 & $14.86^{* * *}$ & .265 & $3.86^{* * *}$ \\
\hline & Professionals or physicians ${ }^{2}$ & .327 & .107 & .037 & $8.02 * *$ & .214 & $3.06 * *$ \\
\hline & Cognitive delays ${ }^{4}$ & .375 & .128 & .021 & $6.11 * *$ & -.198 & $2.83 * *$ \\
\hline \multirow[t]{5}{*}{ Generalization } & Parenting seminar ${ }^{3}$ & .319 & .102 & .102 & $22.39 * * *$ & .267 & $3.92 * * *$ \\
\hline & $\geqq$ University degree $^{1}$ & .375 & .140 & .038 & $8.74 * *$ & .182 & $2.74 * *$ \\
\hline & Professionals or physicians ${ }^{2}$ & .408 & .166 & .026 & $6.19 *$ & .169 & $2.58 * *$ \\
\hline & Cognitivedelays ${ }^{4}$ & .434 & .188 & .022 & $5.21 *$ & -.148 & $-2.30 *$ \\
\hline & Eastern Taiwan $^{5}$ & .454 & .206 & .018 & $4.26^{*}$ & -.134 & $-2.08 *$ \\
\hline \multirow[t]{4}{*}{ Socialization } & Professionals or physicians ${ }^{2}$ & .211 & .045 & .045 & $9.20 * *$ & .201 & $3.02 * *$ \\
\hline & Emotional delays ${ }^{6}$ & .275 & .076 & .031 & $6.54 *$ & -.198 & $-2.96 * *$ \\
\hline & Eastern Taiwan ${ }^{5}$ & .324 & .105 & .029 & $6.37 *$ & -.197 & $-2.87 * *$ \\
\hline & Parenting seminar ${ }^{3}$ & .357 & .128 & .023 & $5.04 *$ & .191 & $2.74 * *$ \\
\hline \multirow[t]{4}{*}{ Initiative } & Professionals or physicians ${ }^{2}$ & .364 & .132 & .132 & $30.00 * * *$ & .338 & $5.11 * * *$ \\
\hline & Parenting seminar ${ }^{3}$ & .421 & .177 & .045 & $10.79 * *$ & .243 & $3.64 * * *$ \\
\hline & $\geqq$ University degree $^{1}$ & .456 & .208 & .031 & $7.56^{* *}$ & .159 & $2.42 * *$ \\
\hline & $\begin{array}{l}\text { Activities of daily living } \\
\text { delays }^{7}\end{array}$ & .475 & .225 & .017 & $4.28 *$ & -.133 & $-2.07 *$ \\
\hline \multirow{4}{*}{$\begin{array}{l}\text { Personality } \\
\text { adaptations and } \\
\text { simultaneous } \\
\text { learning }\end{array}$} & Parenting seminar ${ }^{3}$ & .286 & .082 & .082 & $17.61 * * *$ & .231 & $3.35 * *$ \\
\hline & Professionals or physicians ${ }^{2}$ & .357 & .128 & .046 & $10.24 * *$ & .220 & $3.27 * *$ \\
\hline & Eastern Taiwan ${ }^{5}$ & .400 & .160 & .032 & $7.43 * *$ & -.191 & $-2.93 * *$ \\
\hline & $\geqq$ University degree $^{1}$ & .426 & .182 & .022 & $5.09 *$ & .151 & $2.78^{*}$ \\
\hline \multirow[t]{3}{*}{ Gestalt } & Professionals or physicians ${ }^{2}$ & .202 & .041 & .041 & $8.38 * *$ & .177 & $2.62 *$ \\
\hline & Parenting seminar ${ }^{3}$ & .261 & .068 & .027 & $5.74 *$ & .163 & $2.32 *$ \\
\hline & Southern Taiwan ${ }^{5}$ & .308 & .095 & .027 & $5.73 *$ & -.154 & $-2.20 *$ \\
\hline \multirow[t]{3}{*}{ Mastery } & Professionals or physicians ${ }^{2}$ & .221 & .049 & .049 & $10.09 * *$ & .221 & $3.18 * *$ \\
\hline & $\geqq$ University degree $^{1}$ & .298 & .081 & .032 & $7.01 * *$ & .187 & $2.65^{*}$ \\
\hline & Parenting seminar ${ }^{3}$ & .403 & .107 & .026 & $5.69 *$ & .148 & $2.15^{*}$ \\
\hline \multirow{2}{*}{$\begin{array}{l}\text { Behavioral } \\
\text { counseling }\end{array}$} & Professionals or physicians ${ }^{2}$ & .189 & .036 & .036 & $7.30 * *$ & .189 & $2.71 * *$ \\
\hline & Parenting seminar ${ }^{3}$ & .221 & .061 & .025 & $5.41 *$ & .142 & $2.22 *$ \\
\hline \multirow{5}{*}{$\begin{array}{l}\text { Overall } \\
\text { scale }\end{array}$} & Professionals or physicians ${ }^{2}$ & .312 & .098 & .098 & $21.31 * * *$ & .281 & $4.22 * * *$ \\
\hline & Parenting seminar ${ }^{3}$ & .393 & .155 & .057 & $13.26^{* * * *}$ & .264 & $4.02 * * *$ \\
\hline & Eastern Taiwan $^{5}$ & .417 & .174 & .021 & $5.19 *$ & -.186 & $-2.86 * *$ \\
\hline & Southern Taiwan ${ }^{5}$ & .440 & .193 & .020 & $4.76^{*}$ & -.167 & $-2.54 *$ \\
\hline & $\geqq$ University degree $^{1}$ & .463 & .215 & .019 & $4.47 *$ & .142 & $2.12 *$ \\
\hline
\end{tabular}

$R^{2}$ :Coefficient of determination; $\beta$ :Standardized regression coefficient; ${ }^{*} p<.05 .{ }^{*} p<.01 .{ }^{*} * * p<.001$

${ }^{1}$ reference group: mothers who had a high school degree or a lower educational level.

${ }^{2}$ reference group: acquired parenting skills rather than from professionals or physicians.

${ }^{3}$ reference group: acquired parenting skills rather than from parenting seminars.

${ }_{5}^{4}$ reference group: DDC mothers of young children without cognitive delays.

${ }^{5}$ reference group: DDC mothers resided in northern Taiwan.

${ }^{6}$ reference group: DDC mothers of young children without emotional delays.

${ }^{7}$ reference group: DDC mothers of young children without activities of daily living delays. 


\section{Discussion}

Overall, the present study determined that mothers who had 3- to 6-year-old children with developmental delay in Taiwan had generally exhibited satisfactory parenting skills. The results showed that for DDC mothers, the parenting skill of meeting the basic needs (including home safety, good parent-child relationships, physical needs e.g., food, drink, or sleep, prevention sick, and physical and mental health) of their children was considered as the first priority.

Gestalt in psychology means wholeness. In Taiwan, a preschool program (physical movement and health, cognition, language, society, emotion, and aesthetics) should be integrated as a whole, instead of being divided into several subjects (Early Childhood Education and Care Act, 2018; Taiwan's Ministry of Education, 2016). The gestalt-based skills (item content such as "When teaching my children, I focus on the learning content as a whole." "When teaching my children, I integrate the learning content with practical activities.") in the present study were related to Taiwanese DDC mothers' compliance with the Early Childhood Education and Care Act. However, among the nine parenting skills, the skill related to the Gestalt learning of DDC mothers was the most unsatisfactory. The result accorded with that of the study by Lo et al. (2018) who investigated parenting skills of typical development children (TDC) mothers in Taiwan. MPSQ is good reliability and construct validity, can help mothers of young children identify the advantage and weaknesses in their parenting skills and accordingly provide relevant recommendations for improvement (Lo et al., 2018).Based on the distinguishing criteria of parenting skill published by Lo et al. (2018), this study examined nine parenting skills in the DDC mothers. These mothers had low to medium performance levels in all parenting skills except satisfying basic needs, in which they demonstrated a medium level of skill.

There were no significant differences between mother ages, young children ages and sex for nine and overall parenting skills of DDC mother. This result conformed to the study by Lo et al. (2018).In the present study, the generalization, socialization, personality adaptations and simultaneous learning, Gestalt learning, and overall parenting skills of DDC mothers differ with respect to the urban-rural gap (northern Taiwan was significantly better than eastern Taiwan or southern Taiwan). Taiwan survey showed that parenting skills of TDC mothers in northern Taiwan were significantly higher than those of mothers in eastern Taiwan (Lo et al., 2018).An urban-rural divide results from varying regional conditions. As the capital of Taiwan, Taipei enjoys more favorable economic, transport, and medical support than cities in southern or eastern Taiwan. Generally, capital cities and their surrounding areas provide more opportunities for education and employment and more favorable medical and transport conditions. Mothers living in northern Taiwan therefore have considerably greater parenting skills compared with their counterparts in southern and eastern Taiwan.

DDC mothers with a higher level of education were found to perform significantly better in meeting children's basic needs, generalization, initiative, personality adaptations and simultaneous learning, and mastery dimensions of parenting skills, as well as perform better overall. Young children of mothers with a higher level of education will less parenting anxiety and less role captivity, given their greater coping resources, more joy and more new life meaning (Nomaguchi \& Brown, 2011). When social and material resources, such as job condition, household earnings, and marital status, are taken into account, differences by education should decrease and may clear away (Nomaguchi \& Brown, 2011). It is trusted that education level deeply affects the ability to be flexible in adjusting to one's original core parenting beliefs or skills, in light of external information; parents with a higher level of education are likely to take more to actively learning new parenting related knowledge or skills (Harkness, Super, \& Keefer, 1992; Lo et al.,2018).

This study revealed that DDC mothers can develop poor parenting skills because of their children's delayed development. Children whose cognitive skills are developmentally delayed tend to fall behind TDC of the same age in reasoning, memorizing, and understanding matters, things, and concepts. Consequently, their mothers have considerably poorer parenting skills in preparation and generalization than mothers of children without cognitive delays. Similarly, because children with emotional delays are likely to experience emotional dysregulation and have poorer social adaptation capacities compared with other counterparts of the same age, mothers of such children perform less favorably in developing their socialization skills. Moreover, children with daily-life difficulties acquire skills such as personal hygiene, dressing and bathing, and eating more slowly than those without such problems. Consequently, their mothers also perform less favorably in developing their active skills.

The research findings revealed that the abilities of DDC parents to satisfy various needs of the children, including physiological, safety, social, respect, and self-actualization needs were significantly lower than TDC parents (Fenning et al., 2011; Goodlin-Jones et al., 2009).

Children with development delay lag behind children with typical development regarding cognition, gross motor, fine motor, and verbal expression abilities. They need special assistance for their eating and sleeping and are highly emotional (Bruder, 1993; Fenning et al., 2011; Tsai, 2009). 
Therefore, the parents need to obtain additional abilities (e.g., feeding skills, arrangement of daily schedules, safety protection, prepare suitable teaching materials, cultivate independence, autonomy, and life skills in children) to satisfy the children's needs. China survey ( $\mathrm{Ma}$ et al., 2012) found that elementary school parents' utilization of parenting deliveries, ranked from high to low, were TV/film/broadcasting (64.2\%), books/magazines/newspapers (55.3\%), family/friends (44.5\%), schools (40.2\%), Web (28.3\%), and expert lectures (21.8\%). Taiwan survey found that the information sources of parenting skill of TDC mother, 60\%, 48.2\%, 46.9\%, 30.4\%, 29.5\%, 26.7\%, and 3.9\% came from relatives and friends, newspapers or magazines, Internet, professionals or physicians, TV media, parenting seminars, and broadcasts, respectively (Lo et al., 2018). This study revealed that DDC mothers' utilization of parenting information sources, ranked from high to low, were relatives and friends (56.8\%), professionals or physicians $(52.3 \%)$, Internet (38.2\%), newspapers or magazines (36.7\%), parenting seminars $(29.1 \%)$, TV media (23.1\%), and broadcasts (4.0\%). The results of this study corresponded with those of Lo (2018). Relatives and friends were the top source from which mothers of both TDC and DDC learn parenting skills. However, an analysis of variance demonstrated that the information provided by relatives and friends did not exhibit noticeable effectiveness on DDC mothers when learning parenting skills. Moreover, the second-ranked source for TDC mothers (i.e., newspapers and magazines) was different from that of DDC mothers (i.e., professionals or physicians). According to Taiwan's The Protection of Children and Youths Welfare and Rights Act(2018) and the Early Intervention Allowance Program for Children with Developmental Delays of the Ministry of Health and Welfare (2018), the social welfare departments of each county or city should provide early intervention services and subsidize the appropriate medical treatment for DDC. Therefore, DDC mothers are more likely to consult with professionals or physicians than are TDC mothers.

\section{Conclusions}

In the present study found that DDC mothers whose parenting skills were acquired from "professionals or physicians" or "parenting seminars" exhibited better performance in meeting basic need, preparation, generalization, initiative, personality adaptation and simultaneous learning, mastery, socialization, Gestalt learning, behavioral counseling, and overall parenting skills, achieving a statistically significant correlation. In addition to the development status of their children, mothers of DDC are substantially affected by the factors of parenting information source, place of residence, and personal education level.As with healthy children, the development process and quality in children with special needs are influenced by an ecological system network centered on the family. In such an ecological system, the microsystem that relates to one's family gradually expands to a mesosystem affecting the mother's parenting skills and subsequently can be considered in the context of exosystems and macrosystems. Therefore, the quality of children's development is not merely determined by how a family nurtures a child's growth but also depends on how the external environment supports a family and helps its parents to optimize their parenting role. Government agencies are recommended to fulfill reporting and referral of children with developmental delay and provide early interventions to children with developmental delay. The interventions include professional medical rehabilitation, special education, family support, parenting skill consultation, and welfare service. These interventions can help alleviate developmental delay. Some children who received early intervention attained normal development after intervention, thereby reducing burdens on parents, families, and society.

\section{Acknowledgements}

This study was funded by a grant from the Ministry of Science and Technology, Taiwan (R.O.C.) (Grant No MOST 104-2410-H-142-008-MY2). Author would like to thank the teachers of preschool in Taiwan for their assistance rendered in distribution and collection of questionnaires. Author would like to thank the university student help all data were coded and entered.

\section{References}

Bailey, D. B.,\&Simeonsson, R. J. (1988). Assessing needs of families with handicapped infants.The Journal of Special Education, 22(1), 117-127.

Baker, B. L., McIntyr, L. L., Blacher, J., Crnic, K., Edelbrock, C., \& Low, C. (2003). Preschool children with and without developmental delay: Behaviour problems and parenting stress over time. Journal of Intellectual Disability Research, 47, 217-230.

Baumrind, D. (1967). Child care practices anteceding three patterns of preschool behavior. Genetic Psychology Monographs,75, 43-88.

Borthwick-Duffy, S. A.,\&Eyman, R. K. (1990). Who are the dually diagnosed? American Journal of Mental Retardation, 94, 586-595. 
Bronfenbrenner, U. (1979). The ecology of human development: Experiments by nature and design. Cambridge, MA: Harvard University Press.

Bruder, M. B. (1993). The Provision of early intervention and early childhood special education within community early childhood programs: Characteristics of effective service delivery. Topics in Early Childhood Special Education, 13 (1), 19-37.

Caro, P.,\&Dererensky, J. L. (1991). Family focused intervention model: Implementation and research findings. Topics in Early Childhood Special Education, 11 (3), 66-80.

Chang, S. Y. A. (2014). Correlative study of maternal marital satisfaction and maternal parenting efficiency and parental attitude among urban and rural children (master'sthesis, National Taitung University, Taiwan, R.O.C.).

Chen, Q. H., Joseph, C. Y., \&Wei, Z. J. (2007). Early childhood education and care curriculum design - Theory and practice. Taipei, Taiwan: Donghua.

Cheney, D., Manning, B., \& Upham, D. (1997).Engaging families of students with emotional and behavioral disabilities.Teaching Exceptional children, 30(1), 24-32.

Cheng, Y. (2011). The support and needs of families with infants and young children who had special needs.Kaohsiung Normal University Journal, 31, 1-25.

Chiu, Y. C. (2017). A Study of mothers' parenting attitudes and perspectives on play behaviors towards children of different genders (master's thesis, National Taichung University of Education, Taiwan, R.O.C.).

Cormany, E. E. (1992). Meeting the needs of parent of preschool handicapped children through increased support services(ERIC Document for Research Service No. ED 354671.)

Dettemer, P., Thurston, L. P., \&Dyck, N. (1993).Consultation, collaboration and teamwork for students with special needs.Boston: Allyn and Bacon.

Early Childhood Education and Care Act. Retrieved (2018.11.20) from http://edu.law.moe.gov.tw/EngLawContent.aspx?Type=E\&id=245

Feldman, M. A., Hancock, C. L., Rielly, N., Minnes, P., \& Cairns, C. (2000).Behaviour problems in young children with or at risk for developmental delay.Journal of Child and Family Studies, 9, 247-261.

Fenning, R. M., Baker, B. L., \& Juvonen, J. (2011). Emotion discourse, social cognition, and social skills in children with and without developmental delays. Child Development, 82(2), 717-731.

Fingerman, K. L., Cheng, Y. P., Wesselmann, E. D., Zarit, S., Furstenberg, F., \&Birditt, K. S.(2012). Helicopter parents and landing pad kids: Intense parental support of grown children. Journal of Marriage and the Family, 74(4), 880-896.

Goodlin-Jones, B., Tang, K., Liu, J., \& Anders, T. F. (2009).Sleepproblems, sleepiness and daytimebehavior in preschool-agechildren. Journal of Child Psychology and Psychiatry, 50(12),1532-1540. doi: 10.1111/j.14697610.2009.02110.x.

Harkness, S., Super, C. M., \& Keefer, C. H. (1992). Learning to be an American parent: How cultural models gain directive force. In R. D’ndrade\& C. Strauss (eds.), Human motives and cultural models. New York: Cambridge University Press.

Lee, Y. H. (2010). A study of parental education needs for low-income family of children with developmental delay(master's thesis, National Taitung University, Taiwan, R.O.C.).

Li, T. W. (2003). A comparison of parenting style between urban and rural mothers of young children. NTTU Educational Research Journal,14(2), 173-196.

Lindsay, G. \&Strand, S. (2013). Evaluation of the nationalroll-out of parentingprogrammes across England: the parenting early intervention programme (PEIP). BMC Public Health,13(1), 972.

Lo, M. J., Lin, C. K., \&Kuo, B. C. (2018). Educational attainments and parenting related information associated with parenting skills in mothers of preschooler in Taiwan. Journal of Education \& Social Policy, 5(1), 166-185.

Ma, Y. T., Chen, J. Q., Wang, F. M., Xiao, W. Q., Zhang, M., Zhang, W. J., et al. (2012). Parenting skills situation and need assessment among parents of primary school pupils.Beijing Da Xue Xue Bao, 44(3), 364-368.

Maccoby, E. E.,\& Martin, J. A. (1983).Socialization in the context of the family: parent-child interaction, in Handbook of Child Psychology, Paul H. Mussen, ed. New York: John Wiley \& Sons.

Merrell, K.,\& Holland, M. (1997).Social-emotional behaviour of preschool children with and without developmental delays.Research in Developmental Disabilities, 18, 393-405.

Ministry of Health and Welfare.The Early Intervention Allowance Program for Children with Developmental Delays. Retrieved (2018.12.02) from https:file:///C:/Users/ntcu/Downloads/File_167132.pdf

Ministry of the Interior, R.O.C. Department of Household Registration, M. O. I.Retrieved (2018.12.02) from https://www.ris.gov.tw/app/portal/346 
Nomaguchi, K. M.,\& Brown, S. L. (2011). Parental strains and rewards among mothers: The role of education. Journal of Marriage and the Family; 73(3), 621-636. doi:10.1111/j.1741-3737.2011.00835.x.

Ramey, C. T.,\& Ramey, S. L. (1992).Effective early intervention.Mental Retardation, 30(6), 337-345.

Rowe, J., Barnes, M.,\&Sutherns, S. (2013). Supporting maternal transition: continuity, coaching, and control.The Journal of Perinatal Education, 22(3), 145-155.

Salonen, A., Kaunonen, M., Astedt-Kurki, P., Javenpaa, A., Isoaho, H., \&Tarkka, M. (2009).Parenting self-efficacy after childbirth.Journal of Advanced Nursing,65(11), 2324-2336.

Special Needs Education Longitudinal Study.Survey research data archive. Retrieved (2018.12.02) from https://srda.sinica.edu.tw/datasearch_detail.php?id=1143

Steffenburg, S., Gillberg, C., \& Steffenburg, U. (1996). Psychiatric disorders in children and adolsecents with mental retardation and active epilepsy.Archives of Neurology, 53, 904-912.

Steinberg, L., Lamborn, S. D., Dornbusch, S. M., \&Darling, N. (1992). Impact of parenting practices on adolescent achievement: Authoritative parenting, school involvement an encouragement to succeed. Child Development, 63,1266-1281.

Stromme, P.\&Diseth, T. (2000). Prevalence of psychiatric diagnoses in children with mental retardation: Data from a population-based study. Developmental Medicine and Child Neurology, 42, 266-270.

The Protection of Children and Youths Welfare and Rights Act. Retrieved (2018.12.25) from https://law.moj.gov.tw/ENG/LawClass/LawAll.aspx?pcode=D0050001

Taiwan's Ministry of Education.Curriculum outlines for preschool care-taking activities. Retrieved (2017.08.25) from https://www.ece.moe.edu.tw/wpcontent/uploads/2017/03/\%E5\%B9\%BC\%E5\%85\%92\%E5\%9C\%92\%E6\%95\%99\%E4\%BF\%9D\%E6\%B4\% ВB\%Е5\%8B\%95\%Е8\%AA\%B2\%Е7\%A8\%8B\%E5\%A4\%A7\%Е7\%B6\%B1\%E5\%AE\%8C\%E6\%95\%B4\% E4\%B8\%8A\%Е5\%82\%B3\%E5\%90\%AB\%E7\%99\%BC\%E5\%B8\%83\%E4\%BB\%A4\%E7\%89\%88NEW1_\% E9\%A0\%81\%E7\%A2\%BC\%E6\%9B\%B4\%E6\%96\%B0\%E7\%89\%88.pdf

Tsai, S. K. (2009). The effects of the family-oriented parent education program toward parenting abilities and family functions for parents with developmentally delayed children. Journal of Education Studies, 43(1), 107-132

Tsai, S. K. (2002). The effectiveness of parent education program for parents of developmentally delayed young children. Journal of National Taipei Teachers College, 15, 363-386.

Weinberg, S. L.,\& Richardson, M. S. (1981). Dimensions of stress in early parenting.Journal of Counseling and Clinical Psychology, 49, 686-693.

Williams, P. D., Williams, A. R., Lopez, M., \&Tayko, N. P.(2000). Mothers' developmental expectations for young children in the Philippines.Interonal Journal Nursing Studies,37(4), 291-301.

Williamson, L. L. (1997). Parents as teachers of children program (PATCH). Professional School Counseling, 1(2), 7 12.

Yin, M. H. (2010). The influence on home based service for caregivers' rearing patterns for children with developmental delay (master's thesis, Chang Gung University, Taiwan, R.O.C.). 